Ingeniare - Revista Chilena de Ingeniería, vol. 14 Nº 2, 2006, pp. 135-145

\title{
CONTROL PREDICTIVO DE UN ROBOT TIPO SCARA
}

\section{PREDICTIVE CONTROL OF A SCARA ROBOT}

\author{
Oscar Andrés Vivas Albán ${ }^{1}$ \\ Recibido el 4 de noviembre de 2005, aceptado el 30 de mayo de 2006 \\ Received: November 4, 2005 Accepted: May 30, 2006
}

\begin{abstract}
RESUMEN
Este artículo presenta una aplicación eficiente de un control por modelo de referencia sobre un robot de tipo SCARA. El control estudiado es un control predictivo funcional, el que hace uso de un modelo dinámico simplificado del robot. Los ensayos simulados se realizan sobre un robot de cuatro grados de libertad, tipo SCARA. Con el fin de comparar diferentes estrategias de control, se diseña un controlador clásico tipo PID y dos controladores basados en el modelo de referencia. En este último caso el sistema se linealiza y se desacopla por realimentación, lo que transforma el sistema a controlar en un simple par de integradores. Al sistema lineal y desacoplado resultante se le aplica el control por par calculado y el control predictivo funcional. Los tres controladores estudiados se simulan sobre el robot SCARA con valores numéricos reales. Las pruebas permiten valorar las respuestas de estos controladores en seguimiento de trayectoria, rechazo de perturbaciones y presencia de errores en el modelado con consignas complejas similares a las utilizadas en procesos de fabricación.
\end{abstract}

Palabras clave: Robots industriales, control de robots, control por modelo de referencia, control predictivo funcional.

\begin{abstract}
This paper describes an efficient approach for model based control, applied on a SCARA robot. The studied control is the predictive functional control which uses a simplified dynamical model of the robot. The simulated tests are made on a SCARA type robot, with four DOF. To compare several control strategies, a classical PID control and two model based controllers are designed. In the last case, the model is first linearized and decoupled by feedback, transforming the system into a double set of integrators. Computed torque control and predictive functional control are applied to the linear and decoupled system. The three studied controllers are simulated on the SCARA robot with real numerical values. Tracking performance; disturbance rejection and model robot mismatch are enlightened, using complex machining tasks trajectories and error presence in the modelling with complex features.
\end{abstract}

Keywords: Industrial robots, robot control, model based control, predictive functional control.

\section{INTRODUCCIÓN}

Muchas estrategias han sido utilizadas para el control de robots en los últimos años [1], [2], [3]. Los robots industriales utilizan normalmente el control clásico tipo PID. Pero los brazos manipuladores, los cuales se componen de varias articulaciones unidas entre sí, poseen una dinámica altamente no lineal con un fuerte acoplamiento entre sus respectivas articulaciones. Esto complica la tarea de un simple controlador PID así como su sintonización, sobre todo, con consignas a altas velocidades o aceleraciones. Mejores soluciones han sido entonces propuestas con controladores basados en el modelo del robot, los cuales utilizan un modelo matemático del mecanismo con el fin de compensar los términos dinámicos presentes.

En los brazos robotizados industriales la estrategia de control más común que tiene en cuenta el modelo es el control por par computado [4], [5]. Este control es relativamente fácil de implementar y provee resultados satisfactorios en cuanto a errores de seguimiento y robustez. Pero las incertidumbres presentes en el modelo, debido a parámetros mal estimados o no modelados, dificultan el diseño de un eficaz algoritmo basado en un modelo matemático exacto. Por esto, otros tipos

\footnotetext{
1 Universidad del Cauca, Popayán, Colombia, avivas@unicauca.edu.co
} 
de soluciones han sido diseñados con el fin de darle al sistema la robustez deseada [6], [7].

En la pasada década, el control predictivo (MPCModel Predictive Control) se posicionó como una estrategia efectiva para un gran número de procesos [8], [9]. Muchos trabajos han mostrado que el control predictivo es una opción interesante para el manejo de diversos procesos, al realizarse una optimización del pronóstico del comportamiento del sistema [10], [11], [12]. Este control provee buenas respuestas en términos de rapidez, rechazo de perturbaciones y respuesta frente a errores en los parámetros de la planta. Sin embargo, aunque un significativo número de aplicaciones industriales pueden ser encontradas en procesos químicos o de fabricación de alimentos, donde las dinámicas a tratar son relativamente lentas, pocos resultados pueden encontrarse en el control de procesos no lineales y con alta dinámica, como es el caso de los robots manipuladores [13], [14], donde, además, se manejan órdenes de pocos milisegundos.

Una estrategia especial del control predictivo se presenta en este estudio (control predictivo funcional, [15], [16]), el cual pretende mostrar las ventajas industriales de este tipo de controladores. Esta estrategia será comparada al control por par calculado y al control clásico PID. El robot que se utilizará como planta a controlar es un robot de cuatro grados de libertad tipo SCARA, el cual se conoce y utiliza ampliamente en el mundo industrial y de investigación científica.

Esta investigación se desarrolla con el fin de probar y poner en evidencia las ventajas del control predictivo funcional para el control de brazos manipuladores. Inicialmente se da a conocer la formulación del modelo dinámico del robot seguido por una exposición detallada de la técnica predictiva. Al final se comparará el desempeño de los tres controladores en simulación con el fin de evaluar sus respectivos comportamientos.

\section{FORMULACIÓN DEL MODELO DINÁMICO}

El comportamiento de un brazo robot rígido de $n$ grados de libertad es regido por la siguiente ecuación [3]:

$$
\Gamma=A(\boldsymbol{q}) \ddot{q}+C(\boldsymbol{q}, \dot{\boldsymbol{q}}) \dot{\boldsymbol{q}}+\boldsymbol{g}(\boldsymbol{q})
$$

Donde $\Gamma$ es el par aplicado; $\boldsymbol{A}(\boldsymbol{q})$ es la matriz de inercia, simétrica y definida positiva; $\boldsymbol{C}(\boldsymbol{q}, \dot{\boldsymbol{q}})$ es la matriz que incluye las fuerzas de Coriolis y centrífugas; y $\boldsymbol{g}(\boldsymbol{q})$ es el vector de fuerzas gravitacionales. La posición articular está dada por $\boldsymbol{q}$, la velocidad por $\dot{\boldsymbol{q}}$ y la aceleración por $\ddot{q}$. Esta ecuación dinámica puede también escribirse de manera compacta como:

$$
\Gamma=A(\boldsymbol{q}) \ddot{\boldsymbol{q}}+\boldsymbol{H}(\boldsymbol{q}, \dot{\boldsymbol{q}})
$$

donde $\boldsymbol{H}$ incluye las fuerzas de Coriolis, centrífugas y gravitacionales.

Los términos dinámicos de la Ec. (2) son altamente no lineales y, además, acoplados. El primer paso será entonces linealizar y desacoplar el modelo dinámico del robot. La posibilidad de encontrar dicho linealizador está facilitada por la forma particular del sistema dinámico. En efecto, este es lineal en el control $\Gamma$ y la matriz de rango pleno $\boldsymbol{A}(\boldsymbol{q})$ puede ser invertida para cualquier configuración de robot.

Se propone entonces la siguiente linealización:

$$
\Gamma=\hat{A}(\boldsymbol{q}) \boldsymbol{u}+\hat{\boldsymbol{H}}(\boldsymbol{q}, \dot{\boldsymbol{q}})
$$

donde $\boldsymbol{u}$ representa un nuevo vector de control. $\hat{\boldsymbol{A}} \mathrm{y}$ $\hat{\boldsymbol{H}}$ son las estimaciones de los respectivos términos del robot real. En ausencia de perturbaciones y cuando el modelo dinámico es perfectamente conocido, $\hat{\boldsymbol{A}}=\boldsymbol{A}$ y $\hat{\boldsymbol{H}}=\boldsymbol{H}$. En este caso $\boldsymbol{u}$ tiene la forma de la aceleración articular:

$$
\boldsymbol{u}=\ddot{\boldsymbol{q}}
$$

Esto es equivale a un sistema desacoplado que se representa por un sistema lineal de segundo orden. En otras palabras, el componente $\boldsymbol{u}_{\boldsymbol{i}}$ influencia, con una relación de segundo orden, sólo la variable articular $\boldsymbol{q}_{i}$, independientemente del movimiento de las respectivas articulaciones (figura 1).

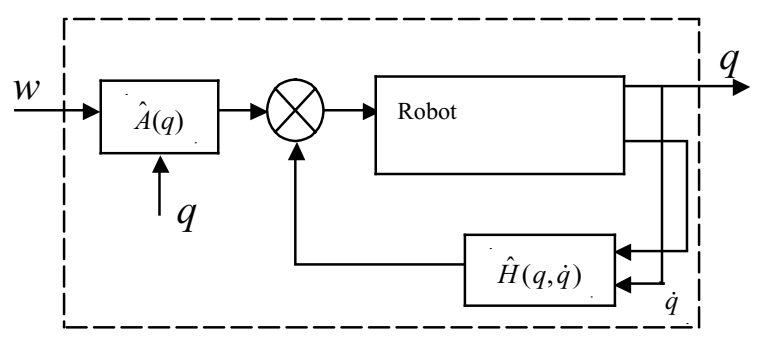

Figura 1. Sistema de linealización por realimentación.

Esta técnica de desacoplamiento y compensación no lineal es muy atractiva desde el punto de vista del control, ya que la dinámica no lineal y con acoplamiento se reemplaza por un sistema lineal de segundo orden y sin acoplamiento. 
Pero la compensación puede no ser perfecta en presencia de incertidumbres en el modelo o debido a las aproximaciones realizadas en el cálculo en línea del modelo inverso.

En la siguiente sección se presentará una técnica basada en el modelo, cuyo fin principal es contrarrestar los efectos de la imperfecta compensación.

\section{CONTROL PREDICTIVO FUNCIONAL}

Esta sección está dedicada a exponer brevemente la teoría del control predictivo funcional (PFC-Predictive Functional Control) usado en esta aplicación, la técnica de control desarrollada por Richalet [15], [16], [17].

\section{Modelo interno}

El modelo utilizado en esta clase de control es un modelo lineal del tipo:

$$
\begin{gathered}
\boldsymbol{x}_{M}(n)=\boldsymbol{F}_{M} \boldsymbol{x}_{M}(n-1)+\boldsymbol{G}_{M} \boldsymbol{u}(n-1) \\
\boldsymbol{y}_{M}(n)=\boldsymbol{C}_{M}{ }^{T} \boldsymbol{x}_{M}(n)
\end{gathered}
$$

donde $\boldsymbol{x}_{M}$ designa el estado, $\boldsymbol{u}$ es la entrada del sistema, $\boldsymbol{y}_{M}$ la salida medida del modelo, $\boldsymbol{F}_{M}, \boldsymbol{G}_{M}$ y $\boldsymbol{C}_{M}$ matrices $\mathrm{y}$ vectores con la dimensión correcta.

Para el caso de sistemas inestables, el control predictivo funcional utiliza una técnica especial llamada de descomposición del modelo [15].

\section{Trayectoria de referencia}

La estrategia del control predictivo MPC se resume en la figura 2. Dada una consigna deseada definida en el horizonte finito $[0, \mathrm{~h}]$, la predicción de la salida futura $\hat{\boldsymbol{y}}_{P}$ deberá alcanzar la consigna futura siguiendo una trayectoria de referencia $\boldsymbol{y}_{R}$.

En la figura 2, $\varepsilon(n)=c(n)-y_{p}(n)$ es el error de posición en el tiempo $n, c$ es la consigna deseada, $y_{P}$ la salida del proceso y TRLC es el tiempo de respuesta del sistema en lazo cerrado, el cual define el horizonte de control. Es claro que este horizonte no debe ser muy pequeño, puesto que aumentará las exigencias del controlador, y no podrá ser muy grande, pues la respuesta del sistema sería demasidado lenta.

La trayectoria de referencia $y_{R}$ está definida por:

$$
c(n+i)-y_{R}(n+i)=\alpha^{i}\left(c(n)-y_{P}(n)\right) \quad 0 \leq i \leq h
$$

donde $\alpha$ es un escalar que se escoge dependiendo del tiempo de respuesta deseado en lazo cerrado.

La esencia de la estrategia de control predictivo está completamente incluida en la Ec. (6). El objetivo es, pues, alcanzar la consigna deseada siguiendo la trayectoria de referencia. Esta trayectoria de referencia se considera entonces como el comportamiento deseado en lazo cerrado.

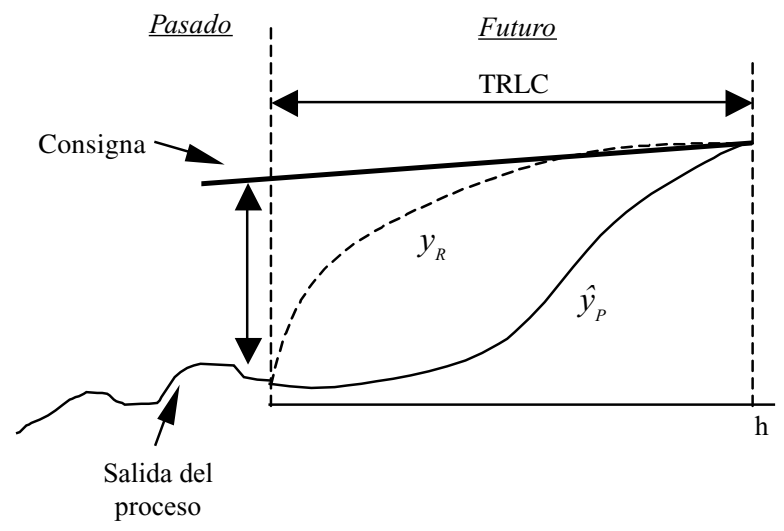

Figura 2. Trayectoria de referencia y estrategia de control.

\section{Índice de desempeño}

El criterio de desempeño se define como la suma cuadrática de los errores entre la predicción de la salida del proceso $\hat{y}_{P}$ y la trayectoria de referencia $y_{R}$. Este criterio se define de la siguiente manera:

$$
D(n)=\sum_{j=1}^{n_{h}}\left\{\hat{y}_{P}\left(n+h_{j}\right)-y_{R}\left(n+h_{j}\right)\right\}^{2}
$$

donde $n_{h}$ es el número de puntos de coincidencia y $h_{j}$ es el número total de puntos de coincidencia definidos sobre el horizonte de predicción. Es en estos puntos de coincidencia que el sistema verificará la aproximación de la predicción de la salida a la trayectoria de referencia. Esta predicción de la salida está definida como:

$$
\hat{y}_{P}(n+i)=y_{M}(n+i)+\hat{e}(n+i) \quad 1 \leq i \leq h
$$

donde $y_{M}$ es la salida del modelo y $\hat{e}$ es la predicción del error de salida futuro.

La estrategia del control predictivo permite introducir un término de suavizamiento al control, con el fin 
de hacer más eficiente el gasto de energía inyectada al sistema. En este caso, el criterio de desempeño se describe como:

$$
D(n)=\sum_{j=1}^{n_{h}}\left\{\hat{y}_{P}\left(n+h_{j}\right)-y_{R}\left(n+h_{j}\right)\right\}^{2}+\lambda\{u(n)-u(n-1)\}^{2}
$$

donde $u$ es la variable de control y $\lambda$ indica el coeficiente de suavizamiento.

\section{Variable de control}

Se supone que la variable futura de control está compuesta por un conjunto de funciones, llamadas funciones de base y que son definidas con anterioridad, lo cual hace que sea más fácil determinar la respuesta futura del sistema. La variable futura de control será entonces:

$$
u(n+i)=\sum_{k=1}^{n_{B}} \mu_{k}(n) u_{B K}(i) \quad 0 \leq i \leq h
$$

donde $\mu_{k}$ son los coeficientes que serán calculados durante la optimización del criterio de desempeño, $u_{B K}$ son las funciones de base de la secuencia de control y $n_{B}$ es el número de funciones de base.

El escogimiento de las funciones de base depende de la naturaleza de la consigna deseada y del proceso. En la práctica ellas son escogidas tan simples como un escalón, una rampa o una parábola y serán definidas como:

$$
u_{B K}=i^{k-1} \quad \forall k
$$

Según la estrategia general del control predictivo, solo el primer término de control se aplica efectivamente, lo que conlleva a:

$$
u(n)=\sum_{k=1}^{n_{B}} \mu_{k}(n) u_{B K}(0)
$$

Por su parte, la salida del modelo está compuesta por dos partes:

$$
y_{M}(n+i)=y_{L}(n+i)+y_{F}(n+i) \quad 1 \leq i \leq h
$$

donde $y_{L}$ es la salida libre del sistema o respuesta a una entrada de control nula $(u=0)$ bajo el efecto de las condiciones iniciales y $y_{F}$ es la salida forzada del sistema en respuesta a la variable de control dada por la Ec. (10) y con condiciones iniciales nulas.

Con las ecuaciones (5) y (10) se puede entonces escribir:

$$
\begin{aligned}
& y_{U F}(n+i)=\boldsymbol{C}_{M}{ }^{T} \boldsymbol{F}_{M}{ }^{i} \boldsymbol{x}_{M}(n) \quad 1 \leq i \leq h \\
& y_{F}(n+i)=\sum_{k=1}^{n_{B}} \mu_{k}(n) y_{B K}(i) \quad 0 \leq i \leq h
\end{aligned}
$$

donde $y_{B K}$ es la respuesta del modelo a $u_{B K}$. Asumiendo que se puede predecir el error de salida futuro del sistema, éste se puede aproximar por el polinomio siguiente:

$$
\hat{e}(n+i)=e(n)+\sum_{m=1}^{d e} e_{m}(n) i^{m} \quad \text { para } 1 \leq i \leq h
$$

donde $d_{e}$ es el grado del polinomio de aproximación del error y $e_{m}$ son coeficientes que se calculan en línea conociendo el error pasado y presente.

Teniendo en cuenta las ecuaciones anteriores, la minimización del índice de desempeño lleva a la siguiente fórmula, la cual muestra el control a aplicarse en el instante presente $n$ :

$$
\begin{aligned}
u(n)= & k_{o}\left\{c(n)-y_{P}(n)\right\}+ \\
& +\sum_{m=1}^{\max (d c, d e)} k_{m}\left\{c_{m}(n)-e_{m}(n)\right\}+V_{X}{ }^{T} x_{M}(n)
\end{aligned}
$$

donde $d_{c}$ es el grado de la aproximación polinomial para la consigna y $k_{0}, k_{m}$ y $V_{X}^{T}$ son coeficientes que se calculan fuera de línea (ver Apéndice).

En resumen, la variable de control que se obtiene está compuesta por tres términos: el primero realiza una compensación proporcional del error de posición, el segundo efectúa una anticipación de la consigna $\mathrm{y}$ del error $\mathrm{y}$ el tercero corresponde a un término de compensación dado que el modelo utilizado no corresponde exactamente al modelo real.

\section{ESTRATEGIAS DE CONTROL}

\section{Descripción del robot SCARA}

El robot SCARA es un brazo manipulador muy utilizado en la industria. Posee cuatro grados de libertad, con tres articulaciones rotatorias y una prismática. Es decir, este robot es capaz de ubicar un objeto en un espacio de tres 
dimensiones, imprimiéndole, además, una rotación, siendo ideal para aplicaciones de ensamblado (Figura 3).

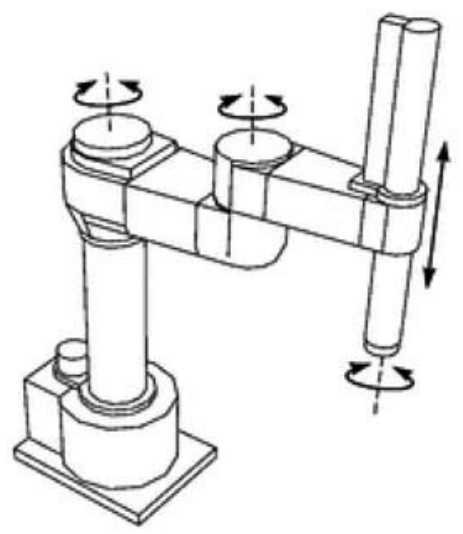

Figura 3. Manipulador tipo SCARA.

La ecuación del modelo dinámico inverso del robot SCARA viene dada por la siguiente expresión Ec. (1) más los términos de frotamientos secos y viscosos, $\boldsymbol{F}_{s j} \mathrm{y}$ $\boldsymbol{F}_{v}$ respectivamente:

$$
\Gamma=\boldsymbol{A}(\boldsymbol{q}) \ddot{\boldsymbol{q}}+\boldsymbol{C}(\boldsymbol{q}, \dot{\boldsymbol{q}}) \dot{\boldsymbol{q}}+\boldsymbol{g}(\boldsymbol{q})+\boldsymbol{F}_{v} \dot{\boldsymbol{q}}+\boldsymbol{F}_{s} \operatorname{sign}(\dot{\boldsymbol{q}})
$$

La matriz de inercia $\boldsymbol{A}$, la matriz que incluye los términos de Coriolis y fuerzas centrífugas $\boldsymbol{C}$ y el vector de gravedad $\boldsymbol{g}$, contienen los parámetros dinámicos del manipulador. Estos parámetros dinámicos son once términos por cada articulación (la matriz de inercia es simétrica): términos del tensor de inercia $\left(\mathrm{XX}_{\mathrm{j}}, \mathrm{XY}_{\mathrm{j}}\right.$, $\mathrm{XZ}_{\mathrm{j}}, \mathrm{YY}_{\mathrm{j}}, \mathrm{YZ}_{\mathrm{j}}, \mathrm{ZZ}_{\mathrm{j}}$ ), primer momento de inercia $\left(\mathrm{MX}_{\mathrm{j}}\right.$, $\left.M Y_{j}, M Z_{j}\right)$, masa de la articulación $\left(M_{j}\right)$ e inercia del accionador $\left(\mathrm{I}_{\mathrm{aj}}\right)$.

Con el fin de aligerar el cálculo de las ecuaciones, los parámetros dinámicos pueden agruparse entre sí, de acuerdo a la determinación de los parámetros de base, obtenidos por medio de consideraciones físicas [5]. Aplicando estas reglas al caso del robot SCARA, se obtienen finalmente los parámetros presentes en las tablas 1 y 2 , donde la letra " $\mathrm{A}$ " indica que el término agrupa valores pertenecientes a otros términos.

Para el robot SCARA, aplicando métodos de identificación paramétrica, se obtienen los valores nominales que se muestran en la tabla 3 [18].

Es de anotar que el modelo dinámico directo se utilizará para simular el brazo robot, mientras que el modelo dinámico inverso [17] se utilizará en la aplicación de las técnicas de control basadas en el modelo, donde se realiza la linealización por realimentación explicada anteriormente.

Tabla 1. Parámetros del tensor de inercia.

\begin{tabular}{|c|c|c|c|c|c|c|}
\hline $\mathrm{j}$ & $\mathrm{XXj}$ & $\mathrm{XYj}$ & $\mathrm{XZj}$ & $\mathrm{YYj}$ & $\mathrm{Yzj}$ & $\mathrm{ZZj}$ \\
\hline 1 & 0 & 0 & 0 & 0 & 0 & $\mathrm{ZZA1}$ \\
\hline 2 & 0 & 0 & 0 & 0 & 0 & $\mathrm{ZZA2}$ \\
\hline 3 & 0 & 0 & 0 & 0 & 0 & $\mathrm{ZZA3}$ \\
\hline 4 & 0 & 0 & 0 & 0 & 0 & 0 \\
\hline
\end{tabular}

Tabla 2. Primer momento de inercia, masas e inercia de los motores.

\begin{tabular}{|c|c|c|c|c|c|}
\hline $\mathrm{j}$ & $\mathrm{MXj}$ & $\mathrm{MYj}$ & $\mathrm{MZj}$ & $\mathrm{Mj}$ & $\mathrm{Iaj}$ \\
\hline 1 & 0 & 0 & 0 & 0 & 0 \\
\hline 2 & MXA2 & MY2 & 0 & 0 & $\mathrm{Ia} 2$ \\
\hline 3 & MXA3 & MYA3 & 0 & 0 & Ia3 \\
\hline 4 & 0 & 0 & 0 & M4 & Ia4 \\
\hline
\end{tabular}

Tabla 3. Valores de los parámetros dinámicos.

\begin{tabular}{|c|c|c|r|}
\hline Parámetro & Valor & Parámetro & \multicolumn{1}{|c|}{ Valor } \\
\hline ZZA1 & 3.38 & Fs1 & 0.57 \\
\hline ZZA2 & 0.063 & Fs2 & 0.125 \\
\hline ZZA3 & 0.1 & Fs3 & 0.05 \\
\hline MXA2 & 0.242 & Fs4 & 0.012 \\
\hline MXA3 & 0.2 & Fv1 & 0.1 \\
\hline MY2 & 0.001 & Fv2 & 0.012 \\
\hline MYA3 & 0.1 & Fv3 & 0.01 \\
\hline Ia2 & 0.045 & Fv4 & 0.001 \\
\hline Ia3 & 0.045 & M4 & 0.5 \\
\hline Ia4 & 0.045 & & \\
\hline
\end{tabular}

Nota: Las unidades para los elementos del tensor de inercia son $\mathrm{Kg} \cdot \mathrm{m}^{2}$, para el primer momento de inercia son Kg.m, para la inercia del accionador Kg. ${ }^{2}$, para los frotamientos secos N.m, para los frotamientos viscosos N.m.s/rad y para las masas Kg.

\section{Control PID}

El controlador PID aplicado al robot SCARA se muestra en la figura 4. Dicho controlador ha sido definido en el espacio operacional, donde las posiciones articulares deseadas son obtenidas a partir del modelo geométrico inverso (MGI). Siguiendo un procedimiento básico de sintonizado [5], las ganancias de los parámetros para cada articulación se fijan en la tabla 4. 
Tabla 4. Ganancias del controlador PID.

\begin{tabular}{|l|r|r|r|r|}
\hline & \multicolumn{1}{|c|}{1} & \multicolumn{1}{c|}{2} & \multicolumn{1}{c|}{3} & \multicolumn{1}{c|}{4} \\
\hline$K_{p}$ & 220.000 & 280.000 & 80.000 & 200.000 \\
\hline$K_{v}$ & 150 & 170 & 120 & 250 \\
\hline$K_{i}$ & 1.000 & 1.000 & 1.000 & 1.000 \\
\hline
\end{tabular}

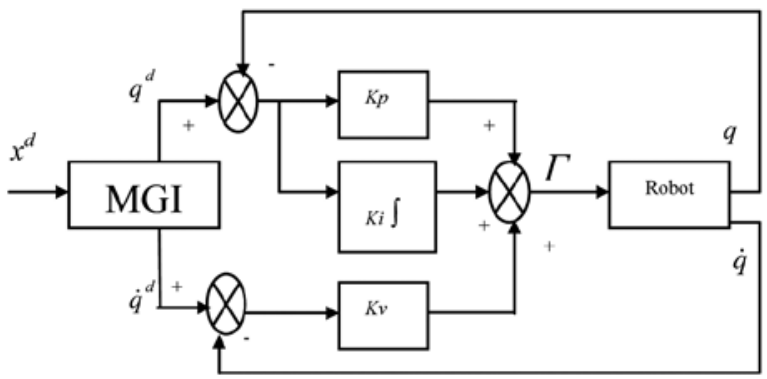

Figura 4. Control PID.

\section{Control por par calculado}

Teniendo en cuenta que el movimiento deseado se especifica con la posición $\boldsymbol{q}^{d}$, el control por par calculado [3], una vez linealizado y desacoplado el sistema como se mostró anteriormente, calcula el vector de control de la siguiente manera:

$$
\boldsymbol{w}=\boldsymbol{K}_{p}\left(\boldsymbol{q}^{d}-\boldsymbol{q}\right)+\boldsymbol{K}_{v} \dot{\boldsymbol{q}}
$$

donde $\boldsymbol{K}_{p}$ y $\boldsymbol{K}_{v}$ representan las ganancias de los controladores. La sintonización de este controlador [5] permite fijar las ganancias en los valores que se muestran en la tabla 5. El esquema de control usado se muestra en la figura 5, donde la consigna deseada se define en el espacio operacional y se transforma en consigna articular gracias al modelo geométrico inverso (MGI).

\section{Control predictivo funcional}

El algoritmo de control predictivo se implementa con la ayuda de un modelo interno compuesto de un doble integrador, el cual representa el sistema no lineal y sin acoplamiento. Para facilitar el proceso de estabilización, dos lazos de realimentación se incluyen en el modelo interno con ganancias que se fijan buscando la mejor respuesta a la salida. En este caso, los lazos de posición y velocidad tienen unas ganancias de $K_{l}=0.01 \mathrm{y}$ $K_{2}=5$ respectivamente, además de un factor de ganancia $G=522$. La ecuación del modelo interno se describe entonces por la función de transferencia en lazo abierto:

$$
G(s)=\frac{522}{s^{2}+1450 s+2.9}
$$

Tres diferentes funciones de base son utilizadas: escalón, rampa y parábola. El tiempo de respuesta en lazo cerrado se fija en 20 veces el tiempo de muestreo $(20 * 1.0 \mathrm{~ms})$, lo cual asegura un buen compromiso entre precisión y robustez de la respuesta. De igual manera, son definidos tres puntos de coincidencia dentro del horizonte de control, ubicados en 4, 13 y $19 \mathrm{~ms}$, ajustados por ensayo y error. El esquema de este controlador se muestra en la figura 6 .

Tabla 5. Ganancias del controlador por par calculado.

\begin{tabular}{|l|r|r|r|r|}
\hline & \multicolumn{1}{|c|}{1} & \multicolumn{1}{c|}{2} & \multicolumn{1}{c|}{3} & \multicolumn{1}{c|}{4} \\
\hline$K_{p}$ & 125.000 & 140.000 & 150.000 & 120.000 \\
\hline$K_{v}$ & 250 & 200 & 1.000 & 700 \\
\hline
\end{tabular}

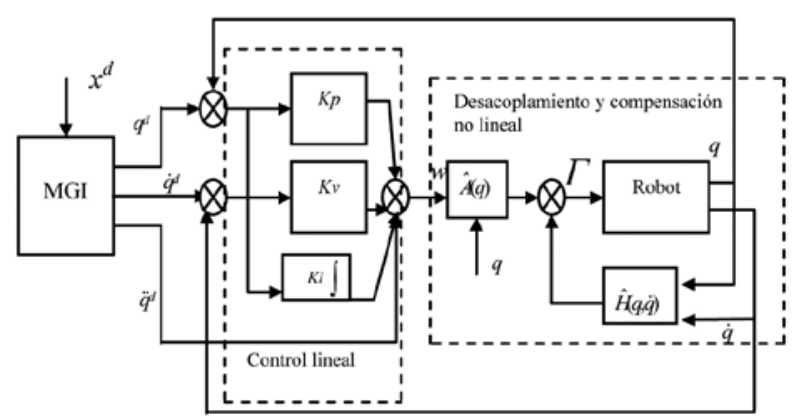

Figura 5. Control por par calculado (CTC).

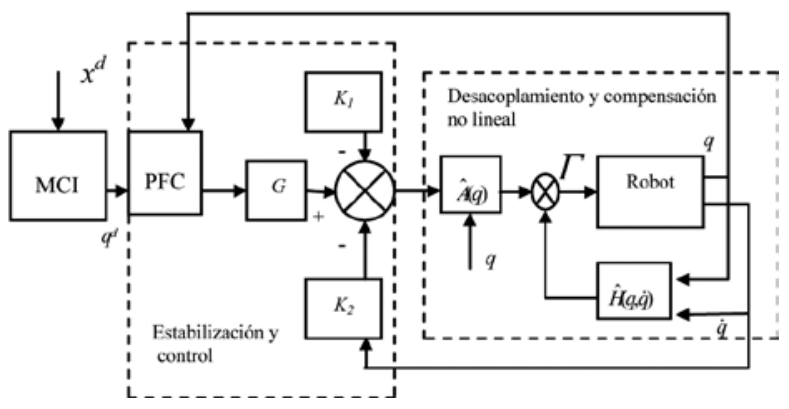

Figura 6. Control predictivo funcional (PFC).

\section{RESULTADOS EN SIMULACIÓN}

El sistema de control se implementa en un simple PC corriendo bajo Windows XP y las simulaciones se llevan a cabo en el ambiente Matlab/Simulink@. Diferentes situaciones se consideran en esta sección con el fin de ilustrar el desempeño de cada controlador. 
Primero, trayectorias complejas definidas en el espacio operacional o cartesiano son utilizadas como consigna. Segundo, se muestran las respuestas a perturbaciones externas, con el fin de probar la robustez de los controladores. Finalmente, a los dos controladores basados en el modelo se les introducen variaciones en los valores de sus parámetros dinámicos para evaluar su robustez frente a estas variaciones.

\section{Desempeño}

Las siguientes trayectorias se definen en el espacio de trabajo o espacio operacional del robot:

(i) Trayectoria circular con diámetro $d=20 \mathrm{~mm}$ y velocidad angular de $\omega=2 \mathrm{rad} / \mathrm{seg}$, lo cual equivale a un círculo realizado en 3 segundos.

(ii) Trayectoria lineal con un cambio de dirección de $55^{\circ}$ y velocidad lineal $v=0.012 \mathrm{~m} / \mathrm{seg}$, equivalente a una trayectoria total realizada en 6 segundos.

Las figuras 7-12 muestran los resultados de la trayectoria circular para los tres controladores, en estado transitorio y estacionario. Los resultados de la trayectoria lineal con cambio de dirección se presentan en las figuras 13, 14 y 15. Para el caso de la consigna circular, los errores máximos en estado transitorio son de $18.5 \times 10^{-3} \mathrm{~m}$ para la respuesta del PID, $2.75 \times 10^{-3} \mathrm{~m}$ para la respuesta del CTC y $1.58 \times 10^{-3} \mathrm{~m}$ para la respuesta del PFC. En el caso estacionario, los errores máximos son $58 \times 10^{-6} \mathrm{~m}$ para la respuesta PID, $5.2 \times 10^{-6} \mathrm{~m}$ para la respuesta CTC y $2.3 \times 10^{-6} \mathrm{~m}$ para la respuesta PFC.

En cuanto a la consigna lineal con cambio de dirección, el error máximo de la respuesta PID es de $12.8 \times 10^{-4} \mathrm{~m}$, de la respuesta CTC es de $4.6 \times 10^{-4} \mathrm{~m}$ y de la respuesta PFC es de $5.2 \times 10^{-4} \mathrm{~m}$.

En esta última prueba (figura 10) se observa que el control por par calculado genera un error ligeramente inferior en un $12 \%$ al generado por el control predictivo funcional. En todos los otros casos es este último cuyos errores de seguimiento son menores.

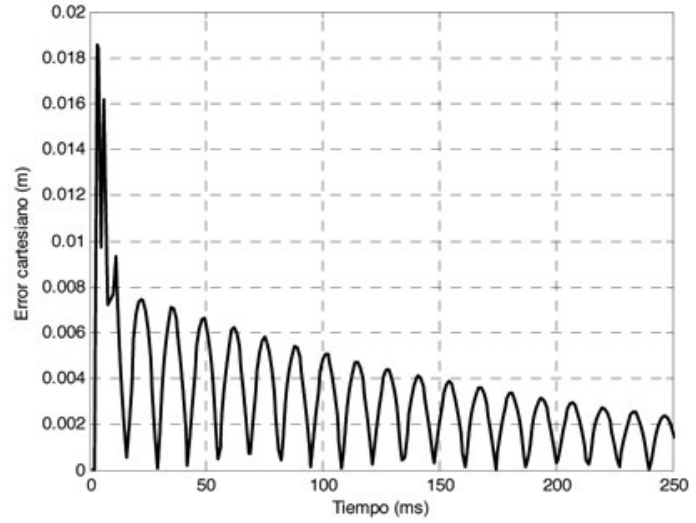

Figura 7. Respuesta transitoria de la consigna circular para el PID.

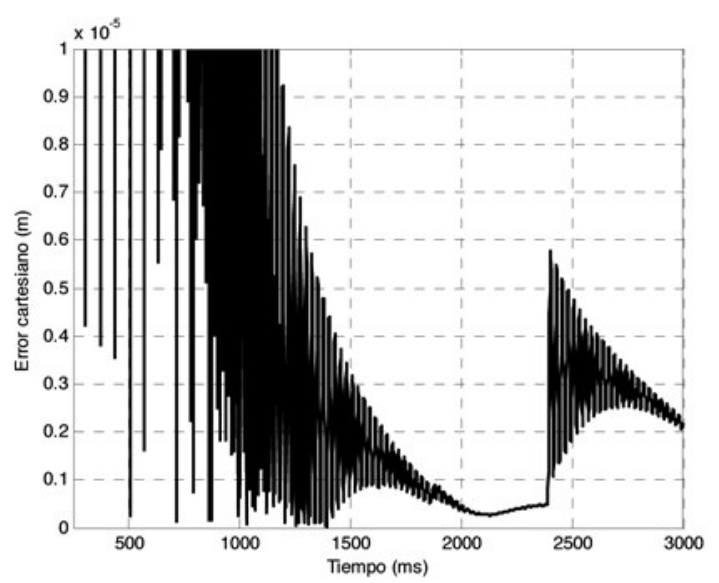

Figura 8. Respuesta estacionaria de la consigna circular para el PID.

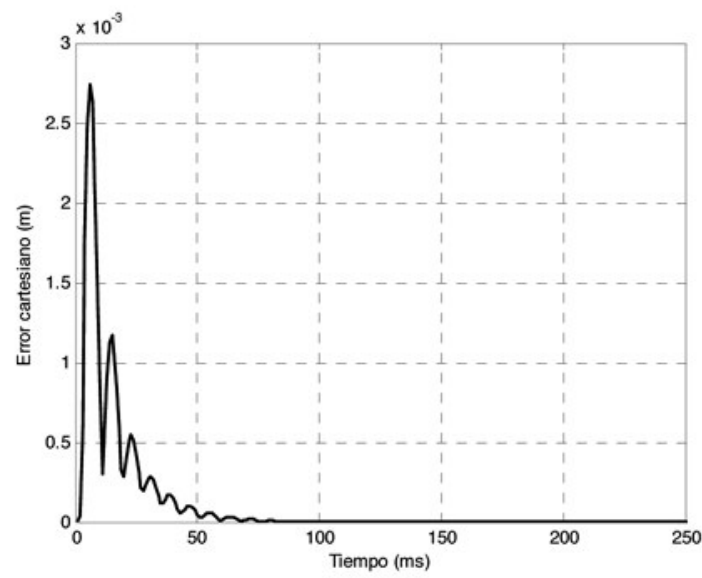

Figura 9. Respuesta transitoria de la consigna circular para el CTC. 


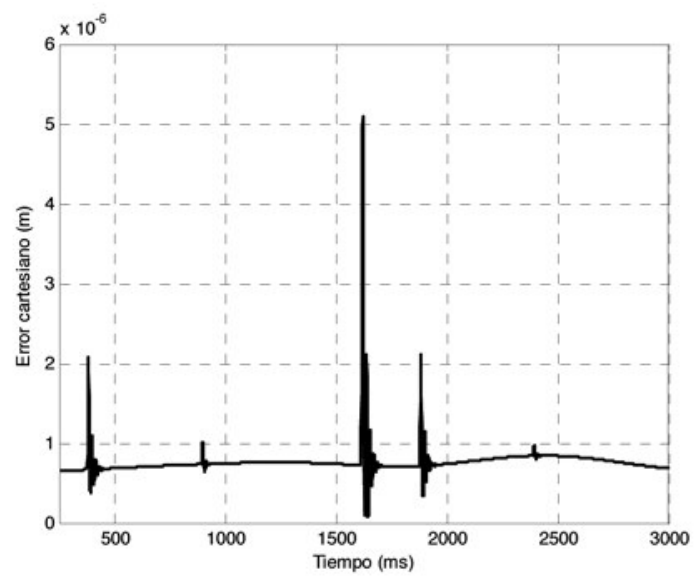

Figura 10. Respuesta estacionaria de la consigna circular para el CTC.

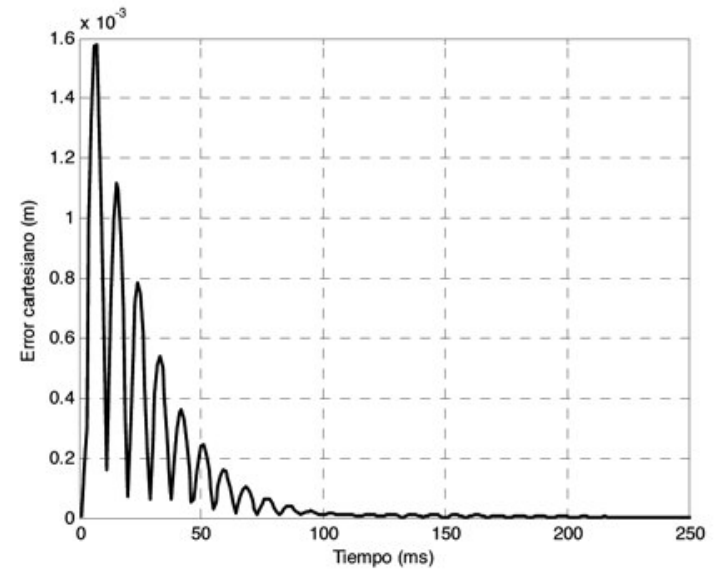

Figura 11. Respuesta transitoria de la consigna circular para el PFC.

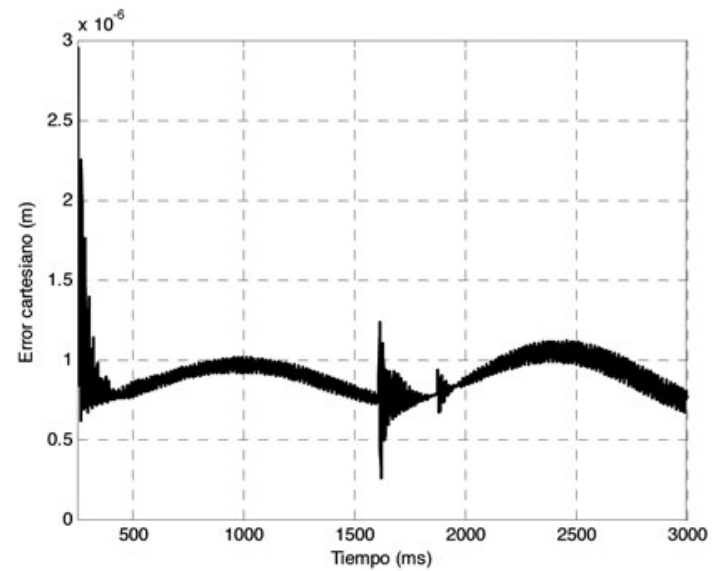

Figura 12. Respuesta estacionaria de la consigna circular para el PFC.

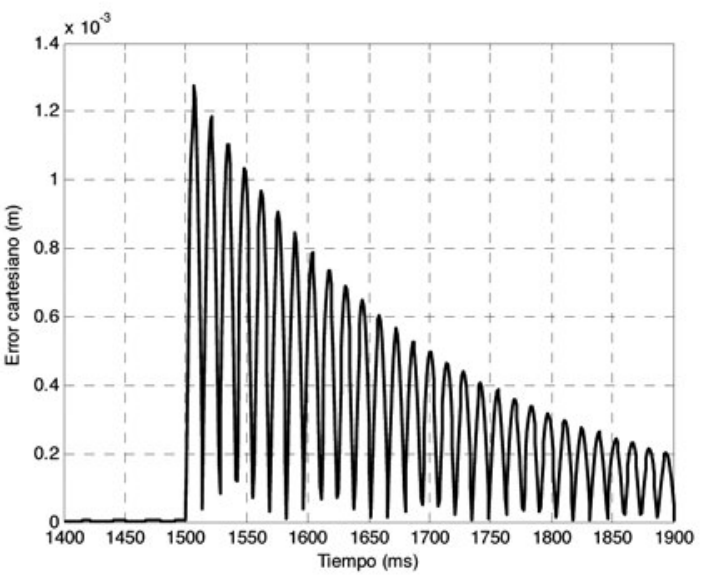

Figura 13. Respuesta ante la consigna lineal con cambio de dirección para el PID.

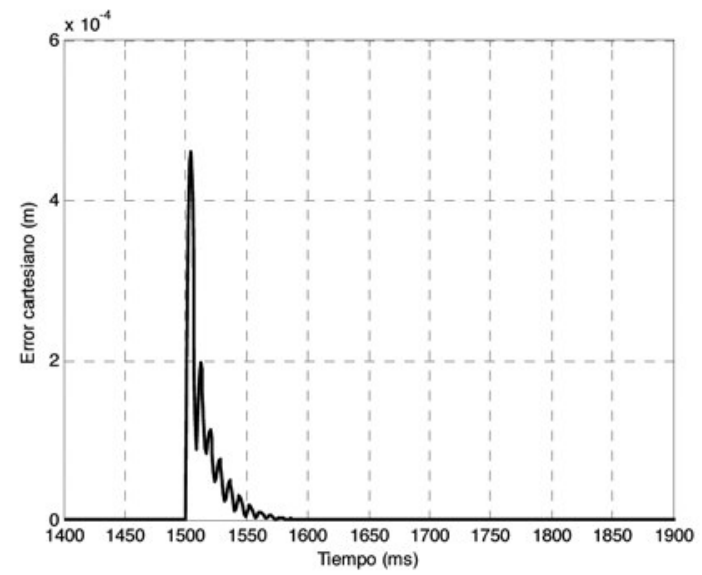

Figura 14. Respuesta ante la consigna lineal con cambio de dirección para el CTC.

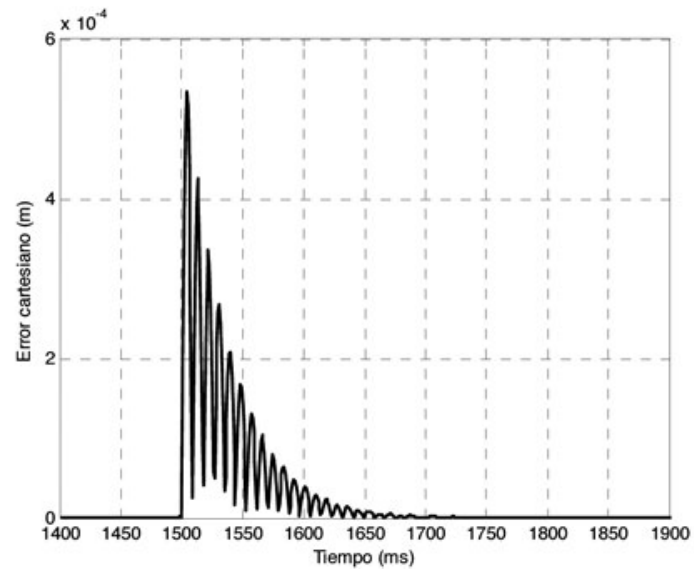

Figura 15. Respuesta ante la consigna lineal con cambio de dirección para el PFC. 


\section{Robustez}

Para probar la robustez, se introduce una perturbación externa al sistema, la cual se define como una variación articular de amplitud 0.08 radianes en un espacio de tiempo reducido. Dado que el sistema se halla en regulación alrededor de una posición estacionaria, el controlador tenderá a absorber esta perturbación con el fin de llevar el sistema lo más rápidamente posible a la posición de reposo inicial.

Las figuras 16, 17 y 18 muestran el rechazo de estas perturbaciones para los controladores PID, CTC y PFC respectivamente. Se observa que el error máximo pico a pico es de alrededor de 8 radianes con fuertes oscilaciones para la respuesta PID, 0.4 radianes para la respuesta CTC y 0.23 radianes para la respuesta PFC.

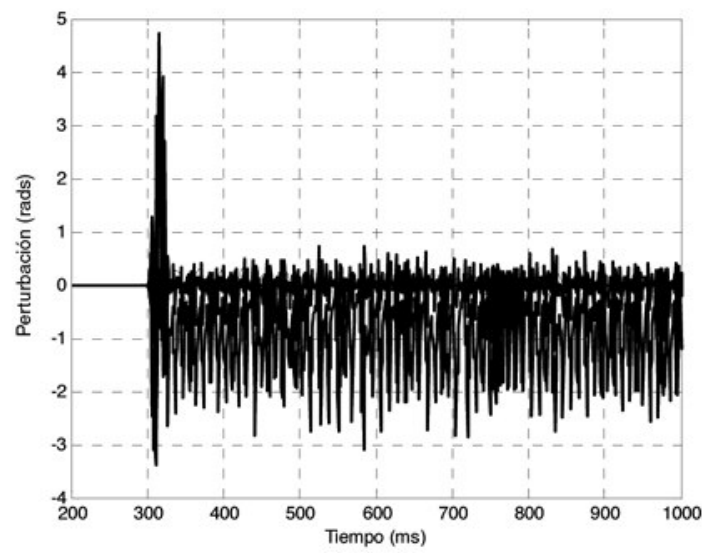

Figura 16. Rechazo de perturbaciones control PID.

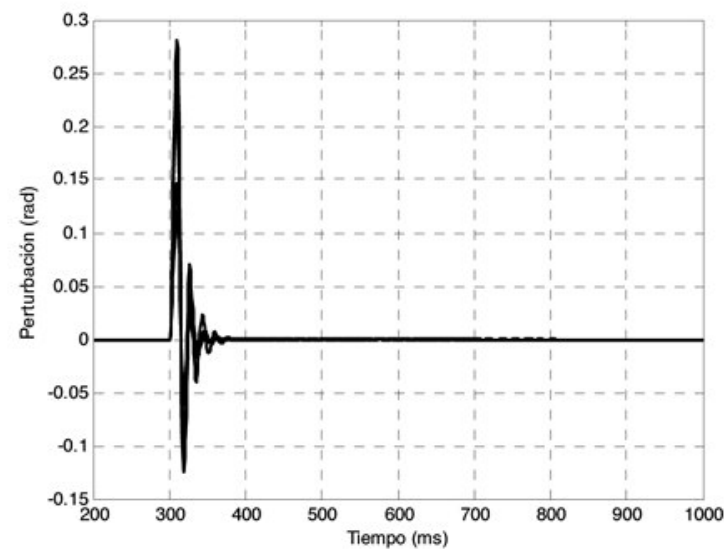

Figura 17. Rechazo de perturbaciones control CTC.

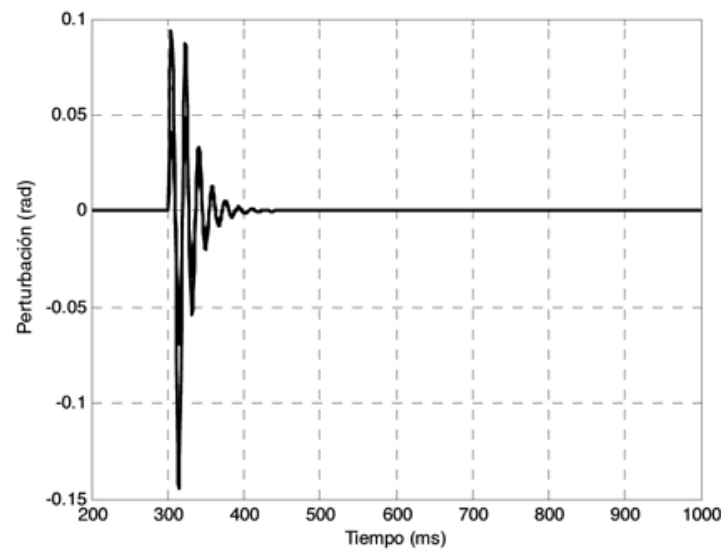

Figura 18. Rechazo de perturbaciones control PFC.

\section{Errores en el modelo}

Para el caso de los dos controladores basados en el modelo dinámico de la planta (control por par calculado y predictivo funcional), se introducen errores en los parámetros dinámicos nominales que se mostraron en la tabla 3. Dichos valores se aumentan hasta un $35 \%$, con lo cual los errores de seguimiento claramente aumentarán para cada controlador, al presentarse una mayor diferencia entre la planta real y la planta estimada.

Es de anotar que errores superiores al $35 \%$ en los valores de los parámetros generan perturbaciones demasiado grandes en las respuestas de los dos controladores, sobre todo en el control CTC.

Se hace entonces de nuevo la prueba con la consigna circular, observándose las respuestas transitoria $\mathrm{y}$ estacionaria para los dos controladores en las figuras 19-22. Para el caso transitorio, los errores aumentan en un $1.200 \%$ en la respuesta del control por par calculado mientras se mantiene prácticamente en su mismo valor para el caso del control predictivo, como se muestra en la tabla 6. De otra parte, los valores máximos de los errores en el caso estacionario son similares a aquellos obtenidos con los valores correctos de los parámetros dinámicos identificados, aunque la respuesta dada por el control CTC con errores en el modelo muestra curvas más bruscas que en el caso sin errores en el modelo.

Tabla 6. Variación de los errores máximos obtenidos al introducirse errores en el modelo.

\begin{tabular}{|l|c|r|r|}
\hline & $\begin{array}{c}\text { Error máximo } \\
\text { sin errores en } \\
\text { el modelo }\end{array}$ & $\begin{array}{c}\text { Error máximo } \\
\text { con errores en } \\
\text { el modelo }\end{array}$ & Variación \\
\hline CTC & $2.75 \times 10^{-3}$ & $33 \times 10^{-3}$ & $+1200 \%$ \\
\hline PFC & $1.58 \times 10^{-3}$ & $1.52 \times 10^{-3}$ & $-4 \%$ \\
\hline
\end{tabular}




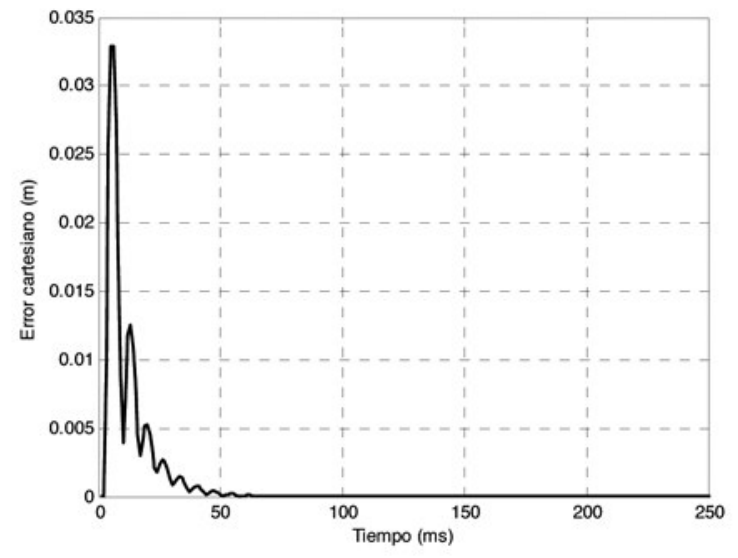

Figura 19. Respuesta transitoria a la consigna circular para el CTC con errores en el modelo.

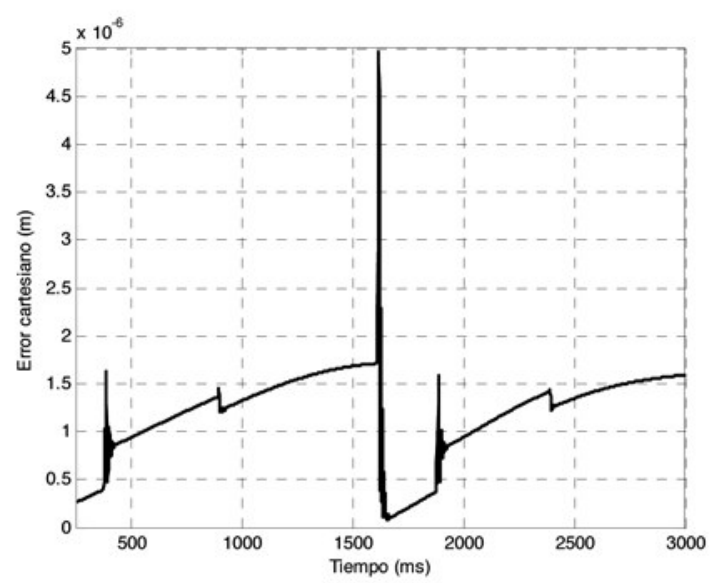

Figura 20. Respuesta estacionaria a la consigna circular para el CTC con errores en el modelo.

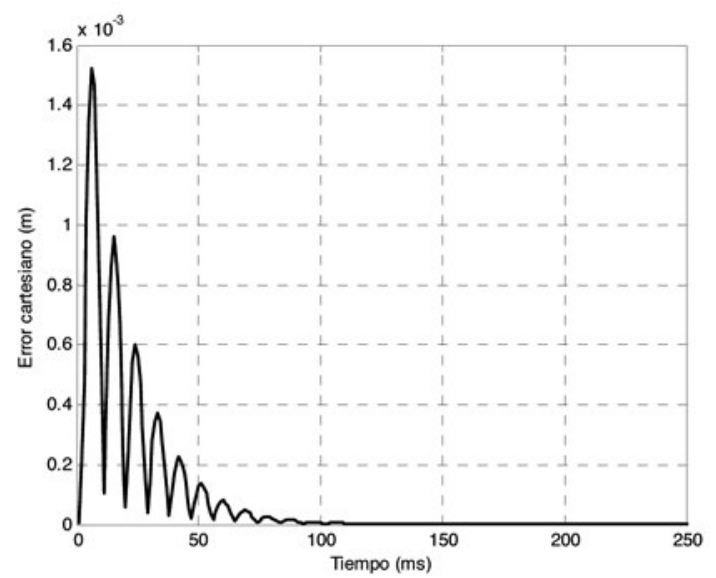

Figura 21. Respuesta transitoria a la consigna circular para el PFC con errores en el modelo.

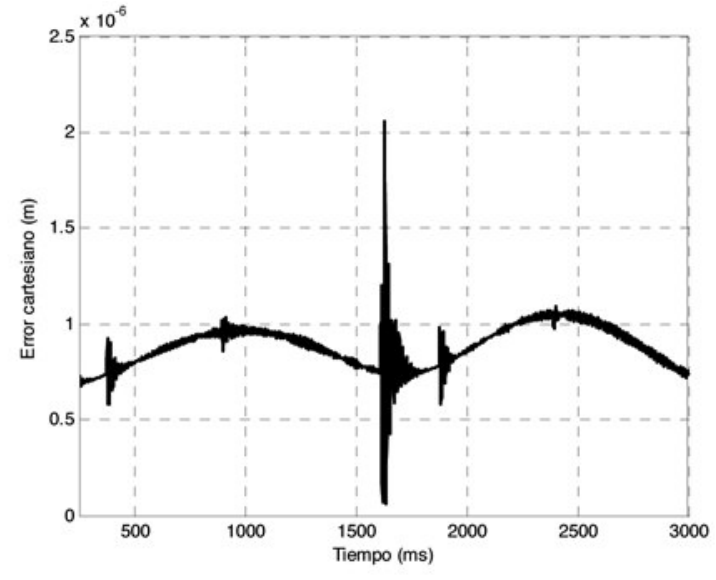

Figura 22. Respuesta estacionaria a la consigna circular para el PFC con errores en el modelo.

\section{CONCLUSIONES}

En este artículo se ha considerado el control avanzado de los movimientos complejos de un robot tipo SCARA.

El desempeño en el seguimiento de consignas complejas, el rechazo de perturbaciones y el comportamiento frente a errores en el modelo son comparados en simulación utilizando tres tipos de controladores: control clásico PID y dos estrategias basadas en el modelo del robot, a saber, el control por par calculado (CTC) y el control predictivo funcional (PFC). Para las dos estrategias basadas en el modelo, el proceso es primero linealizado por realimentación y desacoplado.

Para probar el desempeño de estos controladores se introdujeron trayectorias complejas al sistema (trayectoria circular de pequeño diámetro, trayectoria lineal con cambio de ángulo), similares a aquellas que pueden ser encontradas en un ámbito de fabricación industrial. Igualmente, se realizaron pruebas con el fin de medir la robustez de estos controladores, pruebas consistentes en la introducción de una perturbación externa y para el caso de los dos controladores basados en el modelo, en la introducción de errores en los valores nominales de los parámetros dinámicos. Los resultados muestran que el control predictivo funcional provee los mejores resultados en casi todos los casos, gracias a su capacidad para anticipar los cambios presentes en el sistema. Estos resultados muestran las grandes capacidades que tiene el control predictivo para resolver problemas de control en el campo de la robótica industrial. Futuros trabajos se centrarán sobre la implementación de este controlador sobre un robot SCARA real. 


\section{APÉNDICE}

$\mathrm{k}_{0}=\mathrm{v}^{\mathrm{T}}\left(\begin{array}{c}1-\alpha^{\mathrm{h}_{1}} \\ 1-\alpha^{\mathrm{h}_{2}} \\ \vdots \\ 1-\alpha^{\mathrm{h}_{\mathrm{n}_{\mathrm{h}}}}\end{array}\right), \mathrm{k}_{\mathrm{m}}=\mathrm{v}^{\mathrm{T}}\left(\begin{array}{c}\mathrm{h}_{1}^{\mathrm{m}} \\ \mathrm{h}_{2}^{\mathrm{m}} \\ \vdots \\ \mathrm{h}_{\mathrm{n}_{\mathrm{h}}}^{\mathrm{m}}\end{array}\right), \mathrm{v}_{\mathrm{x}}=-\left[\begin{array}{c}\mathrm{C}_{\mathrm{M}}^{\mathrm{T}}\left(\mathrm{F}_{\mathrm{M}}^{\mathrm{h}_{1}}-\mathrm{I}\right) \\ \mathrm{C}_{\mathrm{M}}^{\mathrm{T}}\left(\mathrm{F}_{\mathrm{M}}^{\mathrm{h}_{2}}-\mathrm{I}\right) \\ \vdots \\ \mathrm{C}_{\mathrm{M}}^{\mathrm{T}}\left(\mathrm{F}_{\mathrm{M}}^{\mathrm{h}_{\mathrm{n}_{\mathrm{h}}}}-\mathrm{I}\right)\end{array}\right]^{\mathrm{T}} \mathrm{v}$

$\mathrm{y} \mathrm{v}=\mathrm{R}^{\mathrm{T}} \mathrm{u}_{\mathrm{B}}(0)$, donde:

$$
\begin{aligned}
& \mathrm{R}=\left\{\begin{array}{llll}
\left.\sum_{j=1}^{\mathrm{n}_{\mathrm{h}}} \mathrm{y}_{\mathrm{B}}\left(\mathrm{h}_{\mathrm{j}}\right) \mathrm{y}_{\mathrm{B}}\left(\mathrm{h}_{\mathrm{j}}\right)^{\mathrm{T}}\right\}^{-1}\left[\mathrm{y}_{\mathrm{B}}\left(\mathrm{h}_{1}\right)\right. & \mathrm{y}_{\mathrm{B}}\left(\mathrm{h}_{2}\right) & \cdots & \mathrm{y}_{\mathrm{B}}\left(\mathrm{h}_{\mathrm{n}_{\mathrm{h}}}\right)
\end{array}\right] \\
& \mathrm{y}_{\mathrm{B}}=\left(\begin{array}{llll}
\mathrm{y}_{\mathrm{B}_{1}} & \mathrm{y}_{\mathrm{B}_{2}} & \cdots & \mathrm{y}_{\mathrm{B}_{\mathrm{B}}}
\end{array}\right)^{\mathrm{T}} \mathrm{u}_{\mathrm{B}}=\left(\begin{array}{lllll}
\mathrm{u}_{\mathrm{B}_{1}} & \mathrm{u}_{\mathrm{B}_{2}} & \cdots & \mathrm{u}_{\mathrm{B}_{\mathrm{B}}}
\end{array}\right)^{\mathrm{T}}
\end{aligned}
$$

\section{REFERENCIAS}

[1] C. Samson, M. Le Borgne, B. Espinau. "Robot control". Oxford University Press. 1991.

[2] F. Lewis, C. Abdallah, D. Dawson. "Control of robot manipulators". Macmillan. 1993.

[3] C. Canudas, B. Siciliano, G. Bastin. "Theory of robot control”. Springer Verlag. 1996.

[4] M. Spong. "Motion control of robot manipulator". Handbook Control. W. Levine Editor, pp. 13391350. CRC Press. 1996.

[5] W. Khalil, E. Dombre. "Modeling identification and control of robots". Hermes Penton Science. 2002.

[6] H. Seraji. "Adaptive Independent joint control of manipulators: Theory and experiment". Proceedings of IEEE International Conference on Robotics and Automation. 1988.

[7] G. Park, D. Hwang. "Robust controller design for a class of nonlinear robot manipulators with actuator dynamics". International Journal of Systems Science. Vol. 33. $\mathrm{N}^{\mathrm{o}}$ 7, pp. 557-565. 2002.
[8] F. Allgöwer, T. Badgwell, J. Qin, J. Rawlings, S. Wright. "Nonlinear predictive control and moving horizon estimation - An introductory overview". Proceedings of the European Conference Control. 1999.

[9] E. Camacho, C. Bordons. "Model predictive control in the process industry". Springer-Verlag. 1995.

[10] D. Clarke, C. Mohtadi, P. Tuffs. "Generalised predictive control". Part. 1: The basic algorithm. Part. 2: Extensions and interpretations. Automatica. Vol. 23. No 2, pp. 137-160. 1987.

[11] P. Boucher, D. Dümur, K. Rahmani. "Generalised Predictive Cascade Control". Proceedings of European Conference Control. 1991.

[12] J. Rossiter. "Predictive functional control: more than one way to prestabilise". Proceedings of the $15^{\text {th }}$ IFAC World Congress. 2002.

[13] P. Poignet, M. Gautier. "Nonlinear model predictive control of a robot manipulator". In proceedings of the $6^{\text {th }}$ International Workshop on Advanced Motion Control. 2000.

[14] A. Vivas, P. Poignet. "Predictive functional control of a parallel robot". Control Engineering Practice. Vol. 13. No 7, pp. 863-874. 2005.

[15] J. Richalet. "Pratique de la Commande Prédictive". Hermès. 1993.

[16] J. Richalet, E. Abu, C. Arber, H. Kuntze, A. Jacubasch, W. Schill. "Predictive functional control - Application to fast and accurate robots". In proceedings of the $10^{\text {th }}$ IFAC World Congress. 1997.

[17] J. Richalet, A. Rault, J. Testud, J. Papon. "Model predictive heuristic control: Applications to industrial process". Automatica. Vol. 14. 1978.

[18] http://www.ladispe.polito.it/robotica/Activities/ Arch/. Departamento de Automática e Informática. Politecnico di Torino. Italia. 